\title{
Association between Metformin Use and Survival in Nonmetastatic Rectal Cancer Treated with a Curative Resection: A Nationwide Population Study
}

\author{
Young-Jun Ki, MD 1 \\ Hyo Jeong Kim, MPH² \\ Mi-Sook Kim, MD, PhD ${ }^{3}$ \\ Chan Mi Park, PhD² \\ Min Jung Ko, $\mathrm{PhD}^{2}$ \\ Young Seok Seo, MD \\ Sun Mi Moon, MD, PhD \\ $\operatorname{Jin} \mathrm{A}$ Choi, $\mathrm{MPH}^{2}$
}

\begin{abstract}
Purpose
Metformin is associated with an anticancer effect. However, the effects of metformin in rectal cancer are controversial. This study investigated the impact of metformin on the survival of patients with diabetes mellitus and nonmetastatic rectal cancer who underwent curative surgery.
\end{abstract}

\section{Materials and Methods}

The database was provided by the Korea Center Cancer Registry and National Health Insurance Service of the Republic of Korea. A cohort of patients with newly diagnosed rectal cancer between 2005 and 2011 was identified. Drug exposure was defined as receiving the oral hypoglycemic agent for at least 90 days over the period from 6 months before the initial diagnosis of rectal cancer to the last follow-up.

\section{Results}

A total of 4,503 patients were prescribed oral hypoglycemic agents and classified as the diabetic group, of which 3,694 patients received metformin for at least 90 days. Unadjusted analyses showed a significantly higher overall survival (hazard ratio, 0.596 ; $95 \%$ confidence interval, 0.506 to 0.702 ) and rectal cancer-specific survival (hazard ratio, $0.621 ; 95 \%$ confidence interval, 0.507 to 0.760 ) in the metformin group than in the nonmetformin group. The adjusted overall survival (hazard ratio, $0.631 ; 95 \%$ confidence interval, 0.527 to 0.755 ) and cancer-specific survival (hazard ratio, $0.598 ; 95 \%$ confidence interval, 0.479 to 0.746 ) in the group with a medication possession ratio of $80 \%$ or greater was significantly higher than in the group with a medication possession ratio of less than $80 \%$.

\section{Conclusion}

Metformin use is associated with overall and cancer-specific survival in diabetic patients with a nonmetastatic rectal cancer treated with a curative resection.
Correspondence: Mi-Sook Kim, MD, PhD Department of Radiation Oncology, Korea Cancer Center Hospital, Korea Institute of Radiological and Medical Sciences,

75 Nowon-ro, Nowon-gu, Seoul 01812, Korea Tel: 82-2-970-1264

Fax: 82-2-970-2412

E-mail: mskim@kcch.re.kr

Co-correspondence: Chan Mi Park, PhD National Evidence-based Healthcare Collaborating Agency, Namasan Square (Kukdong B/D) 7F 173 Toegye-ro,

Jung-gu, Seoul 04554, Korea

Tel: 82-2-2174-2899

Fax: 82-2-747-4916

E-mail: chpark@neca.re.kr

Received March 24, 2016

Accepted June 11, 2016

Published Online July 4, 2016

*Young-Jun Ki and Hyo Jeong Kim contributed equally to this work.
Key words

Rectal neoplasms, Metformin, Diabetes mellitus,

Colorectal surgery, Survival 


\section{Introduction}

Colorectal cancer is the third most common cancer in Korea and worldwide, with a total of 73,759 cancer deaths occurring in Korea during 2012, accounting for $26.7 \%$ of all deaths $[1,2]$. According to the seventh edition of the American Joint Committee on Cancer, the 5-year relative survival rate for stage II and III rectal cancer is $61.6 \%-78.7 \%$ and $31.2 \%-85.1 \%$, respectively [3].

Type II diabetes mellitus (DM) increases the risk of numerous cancer types, including colorectal cancer $[4,5]$. A large cohort study showed that the mortality rate of patients with diabetes is higher than that of patients without diabetes in several cancers [6]. In addition, type $2 \mathrm{DM}$ is associated with a 40\%-60\% increased risk of colorectal cancer [7]. Metformin is the most common antidiabetic drug [8]. Recently, in addition to its antidiabetic effect, it has been shown to reduce the incidence of malignancy and cancer-related mortality $[9,10]$. Several meta-analyses and epidemiologic studies evaluated the effects of metformin on the of incidence cancer, and suggested that metformin is associated with a $20 \%-30 \%$ reduction in carcinogenesis including breast cancers and colorectal cancers $[11,12]$. Inhibition of the mammalian target of the rapamycin pathway has been considered as a mechanism to explain the antitumor effects of metformin [13]. In addition, decreasing the insulin levels in the blood by increasing the insulin sensitivity in patients with diabetes has been considered as a mechanism to explain the antitumor effect of metformin because insulin is considered a tumor growth factor. Although the molecular mechanism describing the antitumor effects of metformin has been elucidated for several years, the effects of metformin in human cancer is an ongoing area of research.

Many studies have shown that in colorectal cancer, metformin is associated with a tumor preventive effect as well as an anticancer effect, reducing mortality $[14,15]$. On the other hand, a few clinical studies reported conflicting results when describing the effects of metformin in colorectal cancer [16]. In the present study, it was hypothesized that the use of metformin might have survival benefits when a homogenous cancer cohort group is analyzed to minimize any selection bias. Although colon and rectal cancer have many similar clinical features, each cancer is treated using different protocols that combine surgery, radiation therapy, and chemotherapy. For rectal cancer, with the exception of early stage cases, the standard treatment is a total mesorectal excision after preoperative chemoradiotherapy [17]. Metformin is associated with a higher pathologic complete response as well as improved survival in patients with rectal cancer receiving preoperative radiotherapy [18]. In contrast, the standard method for colon cancer treatment includes a resection followed by chemotherapy [19]. A study using the Surveillance, Epidemiology, and End Results (SEER) database reported differences in survival between colon and rectal cancer [20]. Therefore, a study of the effects of metformin on the clinical outcomes in the present study cohort focused on patients with pre-existing DM and nonmetastatic rectal cancer treated with a curative resection.

\section{Materials and Methods}

\section{Data source}

The data were initially provided by the Korea Center Cancer Registry (KCCR) and subsequently linked to the national claims data from the National Health Insurance Service (NHIS) of the Republic of Korea, and death registration data from the Korea National Statistical Office, using the personal identification numbers for this study under the consent of the KCCR.

The KCCR data covers cancer cases nationwide from Korea with the inclusion of the date and site of the patients' primary cancer diagnosis [1]. The NHIS covers $100 \%$ of the Korean population, and has comprehensive data sets of the diagnoses, treatments, procedures, surgical history, and prescription records for all insured patients. In addition, the insured population, and their dependents are all required to have periodic general health examinations.

As this study was based routinely on collected administrative data, participant consent was not specifically obtained. This study was approved by the Institutional Review Board of National Evidence-based Healthcare Collaborating Agency (NECA IRB 14-004).

\section{Study subjects}

A cohort of patients with newly diagnosed rectal cancer was identified from the KCCR between 2005 and 2011 (International Classification of Diseases, 10th revision, C20). Individuals aged 18 to 90 years were eligible if they had undergone surgery for rectal cancer with the NHIS. The participants were excluded if they did not undergo surgery for rectal cancer, as well as other criteria including cases of nonadenocarcinoma, SEER stage 7 (distant site[s]/node[s] involved), familial adenomatous polyposis, ulcerative colitis or Crohn disease, synchronous metastasis, death due to complications (death during hospital stay or death due to operative complication), or insulin prescriptions (in the outpatient clinic). Fig. 1 presents the inclusion criteria. 


\section{Exposure and follow-up}

Drug exposure was categorized into metformin or nonmetformin, including sulfonylurea, thiazolidinedione, dipeptidyl peptidase 4 inhibitors, or other oral antidiabetic drugs. Drug exposure was defined as receiving oral hypoglycemic agents (OHA) for at least 90 days during a period 6 months before the initial diagnosis of rectal cancer to the last followup. The medication possession ratio (MPR) was defined as the total number of days the target medication had been prescribed divided by the total number of days between the prescriptions. An MPR greater than $80 \%$ was considered as acceptable adherence. The patient cohort entry dates were the dates when each patient underwent rectal cancer surgery, and the exit date (censoring date) was the earliest date among the date of death, date recording a clinical event, 5 years after cohort entry, or end of the study period (December 31, 2013).

\section{End point}

The primary outcome assessed was the overall survival. The rectal cancer-specific survival data was obtained from the National Population Registry of the Korea National Statistical Office until December 31, 2013, using the unique personal identification numbers.

\section{Covariates}

Information available from the KCCR included data on the patients' cancer diagnosis and date of diagnosis. All other demographic and clinical information was obtained from the claims data or health examination data provided by the NHIS. The cancer-related treatment data, including surgery, radiotherapy, or chemotherapy, as well as the DM-related medications were determined from the NHIS data. Comorbidities occurring from 1 year prior to rectal cancer surgery until the date of surgery were determined from the NHIS claims data.

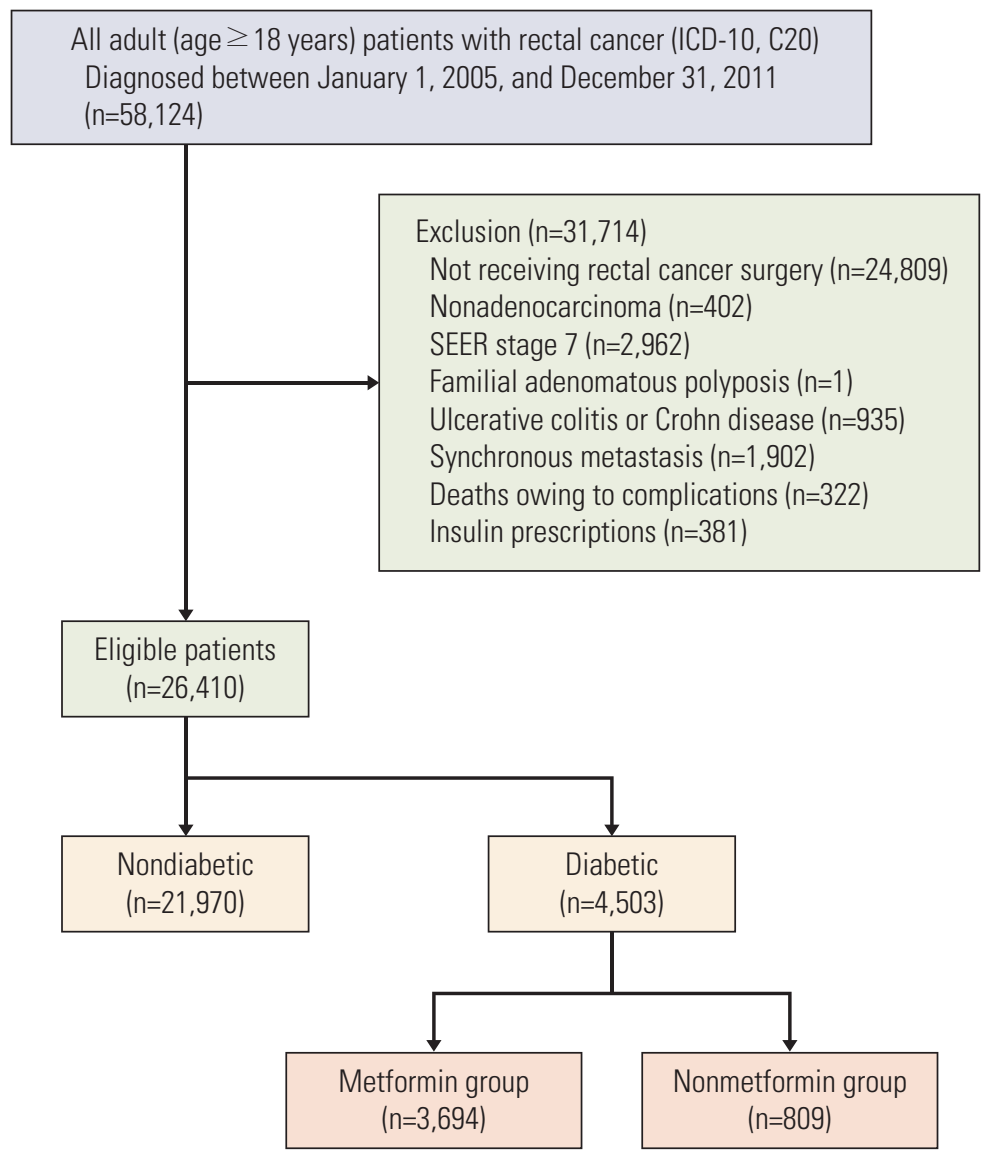

Fig. 1. Flowchart: study population. ICD-10, International Classification of Disease, 10th revision; SEER, Surveillance Epidemiology and End Result. 
Table 1. Baseline characteristics of the rectal cancer patients with diabetes mellitus

\begin{tabular}{|c|c|c|c|}
\hline Characteristic & $\begin{array}{l}\text { Metformin group } \\
\qquad(n=3,694)\end{array}$ & $\begin{array}{l}\text { Nonmetformin group } \\
\qquad(\mathrm{n}=809)\end{array}$ & p-value \\
\hline \multicolumn{4}{|l|}{ Sex } \\
\hline Male & $2,397(64.9)$ & $520(64.3)$ & 0.741 \\
\hline Female & $1,297(35.1)$ & $289(35.7)$ & \\
\hline Age, median (interquartile range, yr) & $65(58-71)$ & $67(60-73)$ & $<0.001$ \\
\hline \multicolumn{4}{|l|}{ SEER stage } \\
\hline Localized & $1,393(37.7)$ & $304(37.6)$ & 0.980 \\
\hline Regional & $2,016(54.6)$ & $444(54.9)$ & \\
\hline Unknown & $285(7.7)$ & $61(7.5)$ & \\
\hline Radiotherapy & $1,322(35.8)$ & $273(33.7)$ & 0.271 \\
\hline Before surgery & $781(21.1)$ & $153(18.9)$ & 0.157 \\
\hline After surgery & $541(14.6)$ & $120(14.8)$ & 0.891 \\
\hline Preoperative chemotherapy & $697(18.9)$ & $137(16.9)$ & 0.200 \\
\hline Adjuvant chemotherapy & $1123(30.4)$ & $216(26.7)$ & 0.037 \\
\hline Charlson comorbidity index score (interquartile range) & $5(4-7)$ & $6(4-7)$ & \\
\hline $0-3$ & $692(18.7)$ & $155(19.2)$ & 0.140 \\
\hline $4-6$ & $1,778(48.1)$ & $356(44)$ & \\
\hline $7-9$ & $976(26.4)$ & $242(29.9)$ & \\
\hline$\geq 10$ & $248(6.7)$ & $56(6.9)$ & \\
\hline
\end{tabular}

Values are presented as number (\%) unless otherwise indicated. SEER, Surveillance Epidemiology and End Result.

A
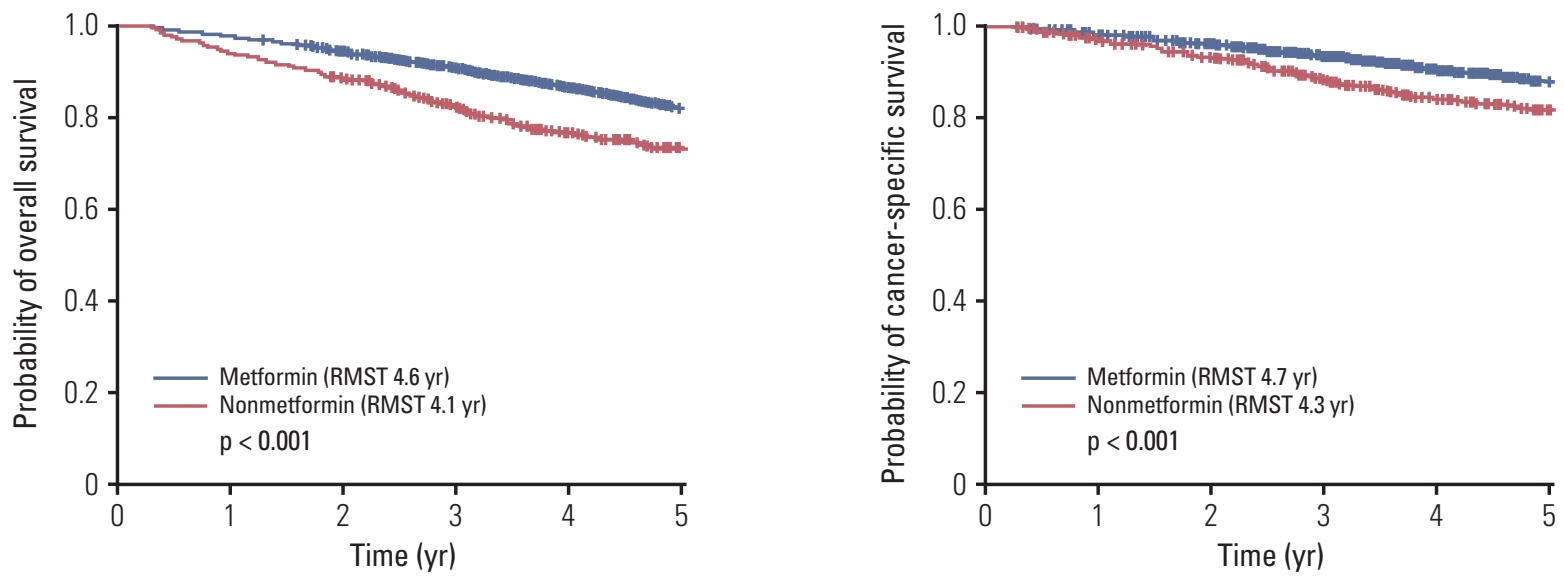

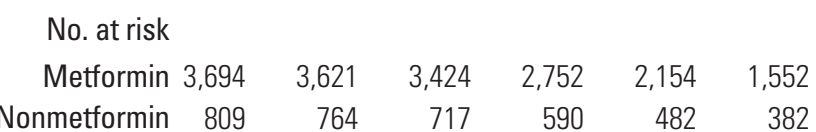

No. at risk

\begin{tabular}{|c|}
\hline nmetformin \\
\hline
\end{tabular}

Fig. 2. Survival curves comparing metformin and nonmetformin use in rectal cancer patients. (A) Overall survival. (B) Rectal cancer-specific survival. RMST, restricted mean survival time. 
Table 2. Mortality risk based on metformin use in patients with rectal cancer

\begin{tabular}{|c|c|c|c|c|c|c|}
\hline \multirow[b]{2}{*}{ Outcome } & \multicolumn{2}{|c|}{ Incidence } & \multicolumn{2}{|c|}{ Unadjusted } & \multicolumn{2}{|c|}{ Adjusted $^{a}$} \\
\hline & $\begin{array}{c}\text { Metformin } \\
\text { group }(n=3,694)\end{array}$ & $\begin{array}{l}\text { Nonmetformin } \\
\text { group }(n=809)\end{array}$ & HR $(95 \%$ CI) & p-value & HR $(95 \%$ CI $)$ & p-value \\
\hline Overall mortality & $541(14.6)$ & $196(24.2)$ & $0.596(0.506-0.702)$ & $<0.001$ & $0.653(0.554-0.770)$ & $<0.001$ \\
\hline RC-specific mortality & $363(9.8)$ & $126(15.6)$ & $0.621(0.507-0.760)$ & $<0.001$ & $0.662(0.540-0.812)$ & $<0.001$ \\
\hline
\end{tabular}

Values are presented as number (\%). HR, hazard ratio; CI, confidence interval; RC, rectal cancer; SEER, Surveillance Epidemiology and End Result. a)Adjusted: sex, age, SEER stage, Charlson's comorbidity index score, preoperative chemotherapy, adjuvant chemotherapy.

\section{Statistical analysis}

The main analysis compared the baseline characteristics and the use of OHA in patients with and without metformin treatment. The rates of overall and rectal cancer-specific survival during the follow-up period among the patients with and without metformin treatment were compared further using the cumulative probability curves derived from Kaplan-Meier estimates. The same analysis was then used to compare the groups based on their MPR. After the unadjusted analyses were initially performed, this study conducted adjusted analyses, including the potential confounders of age, sex, Charlson's comorbidity index [21], SEER stage and adjuvant chemotherapy, anticancer therapy (radiotherapy, chemotherapy) before surgery, and anticancer therapy after surgery. All analyses were performed using SAS ver. 9.3 (SAS Institute Inc., Cary, NC).

\section{Results}

\section{Characteristics of the study population}

Among 58,124 patients with rectal cancer (C20), who were diagnosed between January 1, 2005, and December 31, 2011, 26,410 underwent surgery and met none of the exclusion criteria. Of those patients, 4,503 were prescribed OHAs and were classified as diabetic, with 3,694 receiving metformin for at least 90 days (Fig. 1). The median age of the metformin group was 65 years and that of the nonmetformin group was 67 years. The use of adjuvant chemotherapy was higher in the metformin group than in the nonmetformin group ( $30.4 \%$ vs. $26.7 \%$ ) (Table 1 ).

\section{Clinical outcome}

Among the patients with diabetes during the follow-up period, the overall survival and rectal cancer-specific survival was significantly higher in the metformin group than in the nonmetformin group (Fig. 2). In unadjusted analyses, the metformin group showed a significantly higher overall survival (hazard ratio [HR], 0.596; 95\% confidence interval [CI], 0.506 to 0.702 ) and rectal cancer-specific survival (HR, $0.621 ; 95 \% \mathrm{CI}, 0.507$ to 0.760 ) than the nonmetformin group. After multivariable analysis adjusting for the clinical covariates, the metformin group still had a significantly lower risk of events than the nonmetformin group (Table 2).

In the metformin group, the adjusted overall survival (HR, $0.631 ; 95 \% \mathrm{CI}, 0.527$ to 0.755$)$ and rectal cancer-specific survival (HR, $0.598 ; 95 \%$ CI, 0.479 to 0.746$)$ in patients with an MPR of $80 \%$ or greater, was significantly higher than in the patients with an MPR of less than 80\% (Table 3, Fig. 3).

\section{Discussion}

Several studies have shown that DM increases the mortality of patients with colorectal cancer. Two population-based studies reported that DM is associated with reduced survival in patients with colorectal cancer $[22,23]$. Three meta-analyses reported that patients with diabetes have an increased incidence of mortality from colorectal cancer [24-26]. In addition, Caudle et al. [27] compared the neoadjuvant chemoradiotherapy responses in diabetic and nondiabetic patients with rectal cancer and reported that the complete pathologic response rate was significantly higher in patients without diabetes. In contrast, a case-control study showed no significant difference in survival between patients with colorectal cancer who had well-controlled diabetic diabetes (hemoglobin A1c $<7.5 \%$ ) or who did not have diabetes [7]. 
Table 3. Mortality risk based on the MPR levels in the metformin group

\begin{tabular}{|c|c|c|c|c|c|c|}
\hline \multirow[b]{2}{*}{ Outcome } & \multicolumn{2}{|c|}{ Incidence } & \multicolumn{2}{|c|}{ Unadjusted } & \multicolumn{2}{|c|}{ Adjusted $^{a}$} \\
\hline & $\begin{array}{c}\mathrm{MPR} \geq 80 \% \\
(\mathrm{n}=1,702)\end{array}$ & $\begin{array}{c}\mathrm{MPR}<80 \% \\
(\mathrm{n}=1,992)\end{array}$ & HR $(95 \%$ CI $)$ & p-value & HR $(95 \%$ CI $)$ & p-value \\
\hline Overall mortality & $179(10.5)$ & $362(18.2)$ & $0.606(0.506-0.724)$ & $<0.001$ & $0.631(0.527-0.755)$ & $<0.001$ \\
\hline RC-specific mortality & $116(6.8)$ & $247(12.4)$ & $0.573(0.459-0.714)$ & $<0.001$ & $0.598(0.479-0.746)$ & $<0.001$ \\
\hline
\end{tabular}

Values are presented as number (\%). MPR, medication possession ratio; $\mathrm{HR}$, hazard ratio; $\mathrm{CI}$, confidence interval; $\mathrm{RC}$, rectal cancer; SEER, Surveillance Epidemiology and End Result. a)Adjusted: sex, age, SEER stage, Charlson's comorbidity index score, preoperative chemotherapy, adjuvant chemotherapy.

A

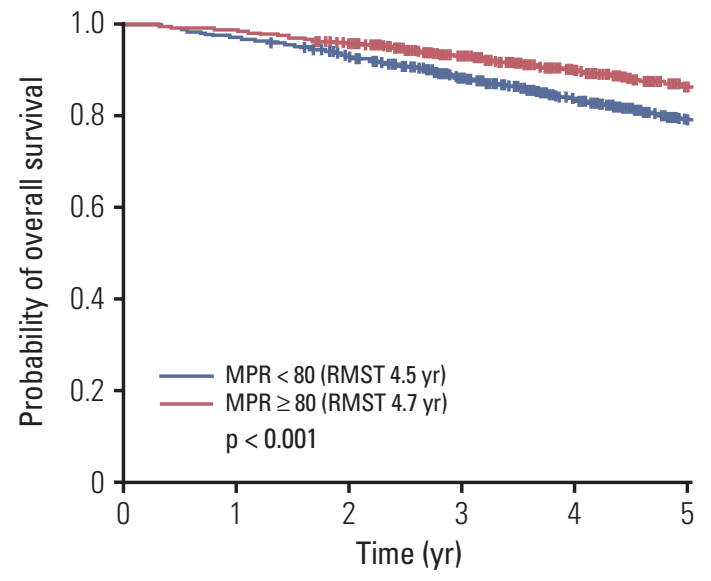

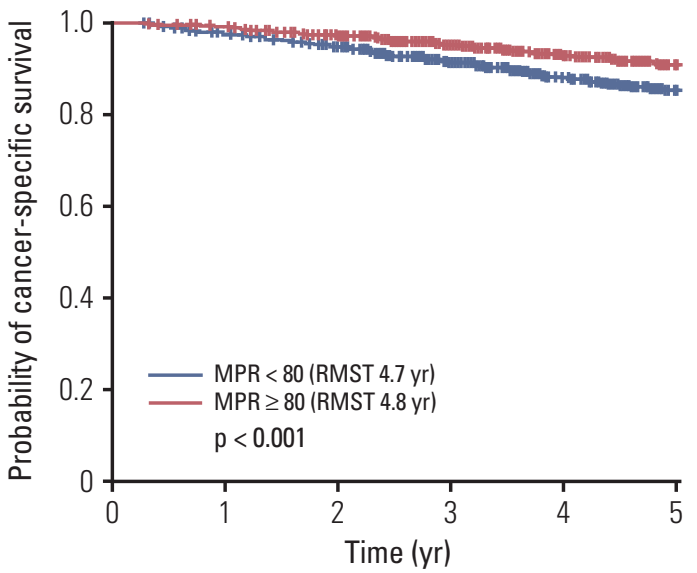

No. at risk

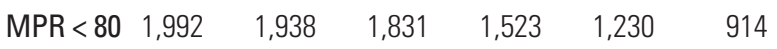

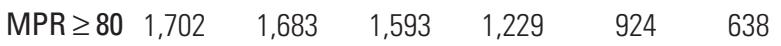

No. at risk

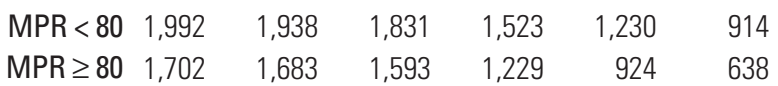

Fig. 3. Survival curves comparing MPR levels for metformin in patients with rectal cancer. (A) Overall survival. (B) Rectal cancer-specific survival. MPR, medication possession ratio; RMST, restricted mean survival time.

Several studies have provided evidence describing a survival relationship between metformin use and colorectal cancer. A single institution study in Korea reported that metformin reduced the overall mortality and colorectal cancer specific mortality in diabetic patients with colorectal cancer [15]. In addition, metformin was reported to have survival benefits compared to other anti-diabetic agents in diabetic patients with colorectal cancer [28]. One meta-analysis showed that patients with colorectal cancer taking metformin had a $34 \%$ improvement in the overall survival compared to nonmetformin users [29]. The only data examining the benefits of metformin focusing on rectal cancer was provided by Skinner et al. [18]. Through a single institute cohort study, the improvement in the pathological complete response rate as well as the outcomes in patients with locally advanced rectal cancer was shown. On the other hand, other studies did not show consistent results describing the beneficial effect of metformin [16]. An Irish study, which compared 207 metformin receiving and 108 nonmetformin receiving patients with stage I-III colorectal cancer and diabetes, reported that the metformin group did not demonstrate a significant difference in survival compared to the nonmetformin group [30].

This study was based on a nationwide population database, which included a large number of patients compared to single institute cohorts. In addition, the analyzed patients included only those with nonmetastatic rectal cancer treated by a curative resection, which is considered a relatively 
homogeneous population with a similar prognosis.

This study has several limitations. First, the patient adherence in taking metformin cannot be confirmed exactly because only the claim data was used. Second, this study could not provide information regarding the appropriate dose of metformin required to observe any anti-tumor effects. The MPR was analyzed for metformin and showed that a higher MPR was associated with improved survival. On the other hand, the optimal dose of metformin could not be provided because the individual dosage data was not available from the NHIS database. The last limitation is the concern that there might be unmeasured confounding factors due to the retrospective design of the study, despite examining a large population. Information on other tumor-related parameters besides tumor stage, such as tumor differentiation, the presence of lymphovascular invasion, or the status of microsatellite instability, was unavailable.

\section{Conclusion}

Metformin use was associated with the overall survival and cancer-specific survival in diabetic patients with nonmetastatic rectal cancer treated by a curative resection. These results could provide evidence for conducting future prospective randomized controlled trials.

\section{Conflicts of Interest}

Conflict of interest relevant to this article was not reported.

\section{Acknowledgments}

This study was supported by the National Evidence-based Healthcare Collaborating Agency, Seoul, Korea (project number NECA-P-14-006).

\section{References}

1. Jung KW, Won YJ, Kong HJ, Oh CM, Cho H, Lee DH, et al. Cancer statistics in Korea: incidence, mortality, survival, and prevalence in 2012. Cancer Res Treat. 2015;47:127-41.

2. International Agency for Research on Cancer. Globocan 2013: cancer fact sheets for colorectal cancer. Lyon: International Agency for Research on Cancer; 2013.

3. Edge SB, Byrd DR, Compton CC, Fritz AG, Greene FL, Trotti A. AJCC cancer staging manual. New York: Springer-Verlag; 2010.

4. Larsson SC, Mantzoros CS, Wolk A. Diabetes mellitus and risk of breast cancer: a meta-analysis. Int J Cancer. 2007; 121:856-62.

5. Friberg E, Orsini N, Mantzoros CS, Wolk A. Diabetes mellitus and risk of endometrial cancer: a meta-analysis. Diabetologia. 2007; 50:1365-74.

6. Coughlin SS, Calle EE, Teras LR, Petrelli J, Thun MJ. Diabetes mellitus as a predictor of cancer mortality in a large cohort of US adults. Am J Epidemiol. 2004;159:1160-7.

7. Siddiqui AA, Spechler SJ, Huerta S, Dredar S, Little BB, Cryer B. Elevated $\mathrm{HbA} 1 \mathrm{c}$ is an independent predictor of aggressive clinical behavior in patients with colorectal cancer: a case-control study. Dig Dis Sci. 2008;53:2486-94.

8. van Staa TP, Patel D, Gallagher AM, de Bruin ML. Glucose- lowering agents and the patterns of risk for cancer: a study with the General Practice Research Database and secondary care data. Diabetologia. 2012;55:654-65.

9. Chowdhury TA. Diabetes and cancer. QJM. 2010;103:90515.

10. Geraldine N, Marc A, Carla T, Chantal M, Stefaan B, Welcome $\mathrm{W}$, et al. Relation between diabetes, metformin treatment and the occurrence of malignancies in a Belgian primary care setting. Diabetes Res Clin Pract. 2012;97:331-6.

11. Decensi A, Puntoni M, Goodwin P, Cazzaniga M, Gennari A, Bonanni B, et al. Metformin and cancer risk in diabetic patients: a systematic review and meta-analysis. Cancer Prev Res (Phila). 2010;3:1451-61.

12. Noto H, Goto A, Tsujimoto T, Noda M. Cancer risk in diabetic patients treated with metformin: a systematic review and meta-analysis. PLoS One. 2012;7:e33411.

13. Dowling RJ, Zakikhani M, Fantus IG, Pollak M, Sonenberg N. Metformin inhibits mammalian target of rapamycin-dependent translation initiation in breast cancer cells. Cancer Res. 2007;67:10804-12.

14. Cardel M, Jensen SM, Pottegard A, Jorgensen TL, Hallas J. Long-term use of metformin and colorectal cancer risk in type II diabetics: a population-based case-control study. Cancer 
Med. 2014;3:1458-66.

15. Lee JH, Kim TI, Jeon SM, Hong SP, Cheon JH, Kim WH. The effects of metformin on the survival of colorectal cancer patients with diabetes mellitus. Int J Cancer. 2012;131:752-9.

16. Cossor FI, Adams-Campbell LL, Chlebowski RT, Gunter MJ, Johnson K, Martell RE, et al. Diabetes, metformin use, and colorectal cancer survival in postmenopausal women. Cancer Epidemiol. 2013;37:742-9.

17. National Comprehensive Cancer Network. NCCN guidelines for treatment of cancer by site: rectal cancer. v2. Fort Washington, PA: National Comprehensive Cancer Network; 2015.

18. Skinner HD, Crane CH, Garrett CR, Eng C, Chang GJ, Skibber $\mathrm{JM}$, et al. Metformin use and improved response to therapy in rectal cancer. Cancer Med. 2013;2:99-107.

19. National Comprehensive Cancer Network. NCCN guidelines for treatment of cancer by site: colon cancer, ver. 2. Fort Washington, PA: National Comprehensive Cancer Network; 2015.

20. Lee YC, Lee YL, Chuang JP, Lee JC. Differences in survival between colon and rectal cancer from SEER data. PLoS One. 2013;8:e78709.

21. Charlson ME, Pompei P, Ales KL, MacKenzie CR. A new method of classifying prognostic comorbidity in longitudinal studies: development and validation. J Chronic Dis. 1987;40: 373-83.

22. Gross CP, Guo Z, McAvay GJ, Allore HG, Young M, Tinetti ME. Multimorbidity and survival in older persons with colorectal cancer. J Am Geriatr Soc. 2006;54:1898-904.

23. Polednak AP. Comorbid diabetes mellitus and risk of death after diagnosis of colorectal cancer: a population-based study.
Cancer Detect Prev. 2006;30:466-72.

24. Luo W, Cao Y, Liao C, Gao F. Diabetes mellitus and the incidence and mortality of colorectal cancer: a meta-analysis of 24 cohort studies. Colorectal Dis. 2012;14:1307-12.

25. Stein KB, Snyder CF, Barone BB, Yeh HC, Peairs KS, Derr RL, et al. Colorectal cancer outcomes, recurrence, and complications in persons with and without diabetes mellitus: a systematic review and meta-analysis. Dig Dis Sci. 2010;55:1839-51.

26. Jiang Y, Ben Q, Shen H, Lu W, Zhang Y, Zhu J. Diabetes mellitus and incidence and mortality of colorectal cancer: a systematic review and meta-analysis of cohort studies. Eur J Epidemiol. 2011;26:863-76.

27. Caudle AS, Kim HJ, Tepper JE, O'Neil BH, Lange LA, Goldberg RM, et al. Diabetes mellitus affects response to neoadjuvant chemoradiotherapy in the management of rectal cancer. Ann Surg Oncol. 2008;15:1931-6.

28. Garrett CR, Hassabo HM, Bhadkamkar NA, Wen S, Baladandayuthapani V, Kee BK, et al. Survival advantage observed with the use of metformin in patients with type II diabetes and colorectal cancer. Br J Cancer. 2012;106:1374-8.

29. Mei ZB, Zhang ZJ, Liu CY, Liu Y, Cui A, Liang ZL, et al. Survival benefits of metformin for colorectal cancer patients with diabetes: a systematic review and meta-analysis. PLoS One. 2014;9:e91818.

30. Spillane S, Bennett K, Sharp L, Barron TI. A cohort study of metformin exposure and survival in patients with stage I-III colorectal cancer. Cancer Epidemiol Biomarkers Prev. 2013; 22:1364-73. 